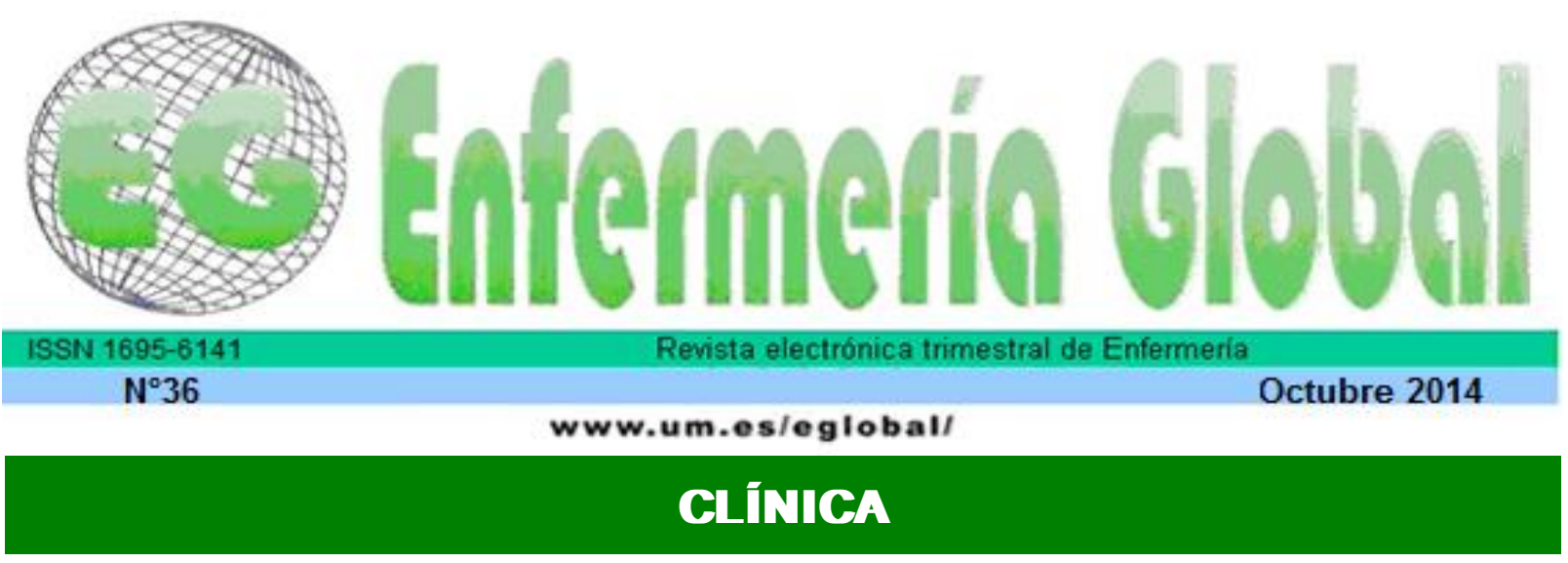

\title{
Adherencia al tratamiento farmacológico y no farmacológico en pacientes con falla cardiaca
}

Adherence to phararmacological and non-pharmacological treatment in patients with heart failure

\section{*Rojas Sánchez, Lyda Zoraya **Echeverría Correa, Luis Eduardo *Camargo}

\section{Figuera, Fabio Alberto}

\begin{abstract}
*Magister em Epidemiología. Grupo de Investigación de Enfermería (GRINFER), Profesor Escuela de Enfermería, Universidad Industrial de Santander, Bucaramanga. E-mail: lydar7@hotmail.com **Médico Internista-Cardiólogo, Clínica de Falla Cardiaca y Trasplante Cardiaco, Fundación
\end{abstract} Cardiovascular de Colombia, Floridablanca, Colombia.

Palabras clave: adherencia al tratamiento; adhesión; cumplimiento de la medicación; falla cardiaca; prevalencia

Keywords: Adherence to treatment; adhesion; medication compliance; heart failure; prevalence

\section{RESUMEN}

Las consecuencias de la no adherencia al tratamiento se expresan en diversas esferas de la vida de la persona, en diversos componentes del sistema de salud y en el cuadro de morbilidad y mortalidad de la población, lo cual genera grandes repercusiones desde el punto de vista médico, económico y en la calidad de vida de la persona.

Objetivo: Determinar la prevalencia de adherencia al tratamiento farmacológico y no farmacológico en pacientes con falla cardiaca.

Métodos: Se realizó un estudio de corte transversal en pacientes con falla cardiaca en el 2012 en la Clínica de Falla Cardiaca y Trasplante Cardiaco de la Fundación Cardiovascular de Colombia. Se incluyeron 161 pacientes adultos con diagnóstico médico de falla cardiaca. Se evaluó la adherencia al tratamiento farmacológico y no farmacológico y el cumplimiento en los pacientes con falla cardiaca.

Resultados: El 80.12\% ( $n=129$; IC 95\% 73.11 - 85.98\%) de los pacientes se encuentra frecuentemente adheridos al tratamiento farmacológico y no farmacológico, el cumplimiento al tratamiento farmacológico con el test de Morisky-Green fue de 53.42\% ( $=86$; IC 95\% 45.39 - 61.3\%).

Conclusiones: Los resultados del presente estudio son un acercamiento a la problemática de la adherencia al tratamiento farmacológico y no farmacológico de los pacientes con falla cardiaca. Se 
recomienda para futuras investigaciones, la realización de estudios multicéntricos con muestreos probabilísticos.

\section{ABSTRACT}

Consequences of non-adherence to treatment are expressed in various spheres of the individual's life, in various components of the health system and in the population's morbidity and mortality, which generates repercussions in the medical and economical aspects and in the individual's life quality.

Objective: To determine the prevalence of adherence to pharmacological and non-pharmacological treatment in patients with heart failure.

Methods: A cross sectional study was conducted in patients with heart failure during 2012 in the Heart Failure and Heart Transplant Clinic of the Fundación Cardiovascular de Colombia. 161 adult patients were included with the diagnosis of heart failure. Adherence to pharmacological and nonpharmacological treatment was evaluated as well as compliance of the patients with heart failure.

Results: $80.12 \%(n=129 ; 95 \% \mathrm{Cl} 73.11-85.98 \%)$ of the patients are often adhered to pharmacological and non-pharmacological treatment, compliance to pharmacological treatment with the Morisky-Green test was of $53.42 \%(n=86 ; 95 \% \mathrm{Cl} 45.39-61.3 \%)$.

Conclusions: The results of this study are an approach to the problem of adherence to pharmacological and non-pharmacological treatment of patients with heart failure. It is recommended for future researches, the realization of multicenter studies with probabilistic samplings.

\section{INTRODUCCIÓN}

La insuficiencia o falla cardiaca (IC) es un importante problema de salud pública; actualmente, sigue siendo una de las condiciones médicas más comunes, costosas, discapacitantes y mortales en todo el mundo ${ }^{1}$. La prevalencia global de la IC es de 20.3 por cada 1000 habitantes y supera los 100 por cada 1000 personas mayores de 65 años. La incidencia anual de la IC es de 1 a 5 por cada 1000 personas y la incidencia relativa se duplica por cada década de vida después los 45 años de edad ${ }^{2}$. El aumento de la prevalencia de la IC refleja un aumento en la incidencia, el envejecimiento de la población y los avances en el tratamiento de las enfermedades cardiacas $^{3}$.

A pesar de los progresos en el tratamiento para la IC, la falta de adherencia al régimen terapéutico prescrito permanece como un problema entre los pacientes con $I^{4}$. La adherencia a las recomendaciones del tratamiento es la principal causa prevenible de re-hospitalización y de mortalidad prematura en los pacientes con $\mathrm{IC}^{5}$. La Organización Mundial de la Salud define la adherencia terapéutica como "el grado en que el comportamiento de una persona tomar el medicamento, seguir un régimen alimentario y ejecutar cambios del modo de vida se corresponde con las recomendaciones acordadas de un prestador de asistencia sanitaria" 6 .

Wu y colaboradores, en una revisión de la literatura de la adherencia a la medicación en pacientes con IC, estiman que la prevalencia de no adherencia a la medicación en pacientes con falla cardiaca oscila entre el $10 \%$ y $93 \%$, la mayoría de los investigadores citan prevalencias alrededor del $40 \%$ y $60 \%$, esto debido a la forma en que se mide y define la adherencia ${ }^{7}$. Las consecuencias de la no adherencia al tratamiento se expresan en diversas esferas de la vida de la persona, en diversos componentes del sistema de salud y en el cuadro de morbilidad y mortalidad de la población, lo cual genera grandes repercusiones desde el punto de vista médico, económico y en la calidad de vida de las personas ${ }^{8}$. 
En nuestro país, la adherencia al tratamiento en pacientes con falla cardiaca ha sido poco estudiada ${ }^{\text {. }}$. Por lo tanto, el objetivo de este estudio fue determinar la prevalencia de adherencia al tratamiento farmacológico y no farmacológico en pacientes que acuden a la Clínica de Falla Cardiaca y Trasplante Cardiaco de la Fundación Cardiovascular de Colombia (CFCTC).

\section{MÉTODOS}

Se realizó un estudio de corte transversal analítico en pacientes con falla cardiaca durante el periodo comprendido entre julio y octubre de 2012 en la CFCTC. Esta es una institución privada de cuarto nivel de atención ubicada en el municipio de Floridablanca-Santander-Colombia, que atiende a pacientes de cualquier régimen de salud, población principalmente de Bucaramanga y su área metropolitana, también acuden personas provenientes de otras regiones del país. Dicha institución contaba en ese tiempo con 406 pacientes activos con falla cardiaca, se consideraba activo el paciente que por lo menos había tenido un control en el último año.

La población de estudio reclutada correspondió a personas mayores de 18 años con diagnóstico médico de falla cardiaca activas de la CFCTC. Personas con alteración en la esfera mental, con alteraciones crónicas o graves que no le permitan responder a la entrevista estructurada y con limitaciones de la comunicación. El protocolo de investigación fue aprobado por el Comité Técnico Científico y el Comité de Ética e Investigación de la Fundación Cardiovascular de Colombia y se realizó el consentimiento informado verbalmente.

El tamaño de la muestra se calculó en StatCalc de Epi Info $6.04 d^{10}$, teniendo en cuenta: un nivel de confianza del 95\%, una población disponible para el estudio de 406 personas activas de la CFCTC, esperando una frecuencia de adherencia al tratamiento del $50 \%$ e intervalos de confianza entre el 45 y $55 \%$, resultando en un tamaño de 198 personas. Este último valor de prevalencia de adherencia al tratamiento esperada se seleccionó de acuerdo al estudio de $\mathrm{Wu}$ et al, quienes reportaron una prevalencia de no adherencia entre el 40 y $60 \%{ }^{7}$. Se realizó un muestreo por conveniencia. Se invitó a participar a todos los pacientes que cumplían los criterios de inclusión, en el momento que ingresaban a la Clínica de Falla Cardiaca para hacer sus consultas de control y se les aplicaba una encuesta tipo entrevista.

La variable dependiente fue la adherencia al tratamiento farmacológico y no farmacológico definida por el "Instrumento de evaluación de adherencia al tratamiento farmacológico y no farmacológico en los pacientes con falla cardiaca"11. El instrumento consta de 10 indicadores y 30 ítems que evalúan los siguientes aspectos; confianza en el profesional de la salud, accesibilidad a los servicio de salud, conocimiento en el manejo del tratamiento farmacológico, aceptación de la enfermedad, reconocimiento de signos y síntomas de alarma, soporte social, mantenimiento del peso, conocimiento de la dieta adecuada, realización de actividad física y estado emocional, este tiene una consistencia interna de 0.72 .

El instrumento cuenta con una escala likert de cuatro alternativas de respuestas donde uno (nunca o nada) significa el valor más bajo de adherencia y cuatro (mucho o siempre) el más alto, donde el valor mínimo que podía obtener un paciente era de 30 y el máximo de 120 puntos. Se consideraba un paciente no adherente con una puntuación entre 30 y 55, poca adherencia entre 56 y 80 , frecuentemente adherente 
entre 81 y 105 y adherido entre 106 y 120 puntos. Se contó con la autorización por escrito de la autora del instrumento para ser utilizado en esta investigación. También se midió el cumplimiento al tratamiento con el Test de Morisky-Green, que valora las actitudes del enfermo con respecto al tratamiento farmacológico ${ }^{12}$.

La persona encargada de la recolección de la información fue una enfermera profesional, quien fue debidamente capacitada en cuanto a la obtención del consentimiento informado, aplicación del instrumento y diligenciamiento del formato de recolección de la información. Antes de iniciar la recolección de la información se realizó una prueba piloto con 10 personas y se realizaron los ajustes necesarios. Se realizó supervisión del trabajo de campo y verificación semanal de la información antes de ser ingresada a las bases de datos, con el objetivo de detectar inconsistencias en la información.

Simultáneamente a la recolección de la información, se construyeron por separado dos bases de datos en EpiData $3.1^{13}$. Se realizó una validación de las mismas para corregir errores, ésta se realizó en el mismo programa. Además, en la construcción de la base de datos se utilizó el subprograma CHECK de EpiData $3.1^{13}$ para restringir la captura de datos y disminuir errores de digitación o de codificación. Luego de corregida la base de datos se exportó para realizar el análisis de la información en el programa estadístico Stata $\mathrm{v} 12^{14}$.

Se realizó un análisis descriptivo de las características de la población de estudio. Las variables en escala de medición cualitativa fueron descritas como frecuencias absolutas y relativas, las variables continuas se describieron con medidas de tendencia central y de dispersión según sus características. Se calculó la prevalencia de la adherencia y sus intervalos de confianza (IC) al 95\%.

\section{RESULTADOS}

\section{Aspectos Generales de la Población Estudio}

\section{Características Sociodemográficas}

Se estudió un total de 161 pacientes, la mediana de edad fue de 66 años con un primer y tercer cuartil de $(Q 1=56 ; Q 3=73)$, una edad mínima de 20 y una máxima de 93 años; la mediana de distancia en tiempo desde el sitio de residencia a la Clínica de Falla cardiaca fue de 60 minutos $(Q 1=15, Q 3=180)$, con una distancia mínima de 5 minutos y una máxima de 960 minutos; la mediana de años escolares cursados y aprobados fue 6 años $(Q 1=3 ; Q 3=11)$, con una mínima de 0 años y una máxima de 16 años (Tabla 1).

Tabla 1. Características Sociodemográficas de Pacientes con Falla Cardiaca $(n=161)$

Variable $\mathrm{n}(\%)$

Sexo

Femenino

Masculino

$97(60.25)$ 


\section{Estado Civil}

Soltero

$8(4.97)$

Casado

$93(57.76)$

Unión libre

$28(17.39)$

Divorciado/Separado

$10(6.21)$

Viudo

22 (13.66)

Soporte de Cuidador

Si

144 (89.44)

No

17 (10.56)

\section{Estrato Socioeconómico}

Bajo

$71(44.10)$

Medio

73 (45.34)

Alto

$17(10.56)$

Área de Residencia

Urbana

$140(86.96)$

Rural

$21(13.04)$

\section{Seguridad Social}

Contributivo

83 (51.55)

Subsidiado

30 (18.63)

Prepagada

$17(10.56)$

Régimen especial

$29(18.01)$

Particular

$2(1.24)$

Ocupación

Empleado

5 (3.11)

Independiente

$21(13.04)$

Desempleado/Cesante

87 (54.04)

Pensionado/Jubilado

$48(29.81)$ 


\begin{tabular}{lc}
\hline Ingresos Mensuales Familiares & \\
0 - 1 S.M.M.L.V & $60(37.27)$ \\
1 - 2 S.M.M.L.V & $38(23.60)$ \\
2 - 4 S.M.M.L.V & $33(20.50)$ \\
4 - 8 S.M.M.L.V & $17(10.56)$ \\
$>$ & 8 S.M.M.L.V \\
No sabe o se rehúsa & $11(6.83)$ \\
\hline salario mínimo mensual legal vigente.
\end{tabular}

\section{Características Clínicas}

La mediana del tiempo de diagnóstico de la falla cardiaca fue de 39 meses $(\mathrm{Q} 1=18$; Q3=72), con un tiempo mínimo de 1 y un máximo de 300 meses; la mediana de fracción de eyección ventricular izquierda fue 25\% (Q1=15;Q3=38) con una fracción mínima del $8 \%$ y una máxima $70 \%$; la mediana del número de hospitalizaciones en el último año fue de 0 veces $(\mathrm{Q} 1=0 ; \mathrm{Q} 3=1)$; el promedio del perímetro abdominal en hombres fue 89.4 con una desviación estándar (DE) de \pm 13.5 y en mujeres fue de $91.6 \mathrm{~cm}$ (DE \pm 10.8$)$ (Tabla 2).

Tabla 2. Características Clínicas de Pacientes con Falla Cardiaca ( $n=161)$

Comorbilidades Presentes

\section{Variable n (\%)}

Enfermedad cerebro vascular

17 (10.56)

Diabetes

$33(20.50)$

EPOC

16 (9.94)

Enfermedad arterial periférica

Insuficiencia renal en diálisis

Hipotiroidismo

28 (17.39)

Enfermedad de Chagas

32 (19.88)

Hipertensión

91 (56.52)

Dislipidemias

$76(47.20)$

Índice Charlson

Ausencia de comorbilidad (0-1 Comorbilidad) 97 (60.25)

Baja comorbilidad (2 Comorbilidades) $\quad 47$ (29.19)

Alta comorbilidad ( $\geq 3$ Comorbilidades) $\quad 17$ (10.56) 


\begin{tabular}{|c|c|}
\hline $\begin{array}{l}\text { Etiología de la Falla Cardiaca } \\
\text { Isquémica }\end{array}$ & $55(34.16)$ \\
\hline Chagásica & $30(18.63)$ \\
\hline Origen desconocido & $29(18.01)$ \\
\hline Valvular & 15 (9.32) \\
\hline Hipertensión & $13(8.07)$ \\
\hline Multifactorial & $9(5.59)$ \\
\hline Otra & $7(4.35)$ \\
\hline Congénita & $1(0.62)$ \\
\hline Hipertensión pulmonar & $1(0.62)$ \\
\hline Multifactorial & $1(0.62)$ \\
\hline $\begin{array}{l}\text { Clasificación Funcional NYHA } \\
\text { I }\end{array}$ & $13(8.07)$ \\
\hline II & $89(55.28)$ \\
\hline III & $54(33.54)$ \\
\hline IV & $5(3.11)$ \\
\hline $\begin{array}{l}\text { Alguna Cirugía Cardiaca } \\
\mathrm{Si}\end{array}$ & $58(36.02)$ \\
\hline No & $103(63.98)$ \\
\hline $\begin{array}{l}\text { Marcapasos } \\
\mathrm{Si}\end{array}$ & $54(33.54)$ \\
\hline No & $107(66.46)$ \\
\hline $\begin{array}{l}\text { Tipo de Marcapasos } \\
\text { Marcapasos }\end{array}$ & $18(11.18)$ \\
\hline Cardiodesfibrilador & $17(10.56)$ \\
\hline Cardioresincronizador & $23(14.29)$ \\
\hline $\begin{array}{l}\text { Alcohol } \\
\mathrm{Si}\end{array}$ & $7(4.35)$ \\
\hline No & 154 (95.65) \\
\hline $\begin{array}{l}\text { Fuma } \\
\mathrm{Si}\end{array}$ & $1(0.62)$ \\
\hline
\end{tabular}




NYHA (New York Heart Association)

\section{Tratamiento Actual para la Falla Cardiaca}

La mediana de pastillas que un paciente toma al día es de 8 pastillas (Q1=7;Q3=10), con un mínimo de 2 y un máximo de 17 pastillas día, en la Tabla 3 podemos observar los medicamentos que recibe el paciente para el manejo de la falla cardiaca.

Tabla 3. Tratamiento Actual de Pacientes con Falla Cardiaca $(n=161)$

\begin{tabular}{lr}
\hline \multicolumn{1}{c}{ Variable } & \multicolumn{1}{c}{$\mathbf{n}(\%)$} \\
\hline IECA & $62(38.51)$ \\
Enalapril & $61(98.39)$ \\
Betabloqueadores & $1(1.61)$ \\
Carvedilol & $153(95.03)$ \\
Bisoprolol & $76(49.67)$ \\
Metoprolol & $53(34.64)$ \\
Nebivolol & $23(15.04)$ \\
ARA II & $1(0.65)$ \\
Losartán & $80(49.69)$ \\
Cardesartán & $37(46.25)$ \\
Valsartán & $35(43.75)$ \\
Irbesartán & $4(5.0)$ \\
Telmisartán & $3(3.75)$ \\
Espironolactona & $1(1.25)$ \\
Furosemida & $111(68.94)$ \\
Hidroclorotiazida & $111(68.94)$ \\
Digoxina & $6(3.73)$ \\
Antiagregantes plaquetarios & $67(41.61)$ \\
Anticoagulantes & $92(57.14)$ \\
& $53(32.92)$ \\
\hline
\end{tabular}


IECA=Inhibidores de la enzima convertidora de angiotensina; ARA II= antagonistas de los receptores de angiotensina II.

\section{Adherencia al Tratamiento Farmacológico y No Farmacológico}

El promedio de adherencia al tratamiento farmacológico y no farmacológico obtenido con el instrumento fue de 89.3 puntos ( $\mathrm{DE} \pm 9.04)$, con un puntaje mínimo de 61 y máximo de 113 puntos. No se encontró ningún paciente en la categoría de no adherente, el $16.15 \%(n=26$; IC $95 \% 10.82-22.75 \%)$ es poco adherente, el $80.12 \%$ $(n=129$; IC $95 \% 73.11-85.98 \%)$ de los pacientes se encuentran frecuentemente adheridos al tratamiento y el $3.73 \%$ ( $n=6$; IC $95 \% 1.38-7.93 \%)$ esta adherido al tratamiento (Figura 1).

Figura 1. Adherencia al Tratamiento Farmacológico y No Farmacológico en Pacientes con Falla Cardiaca n 16

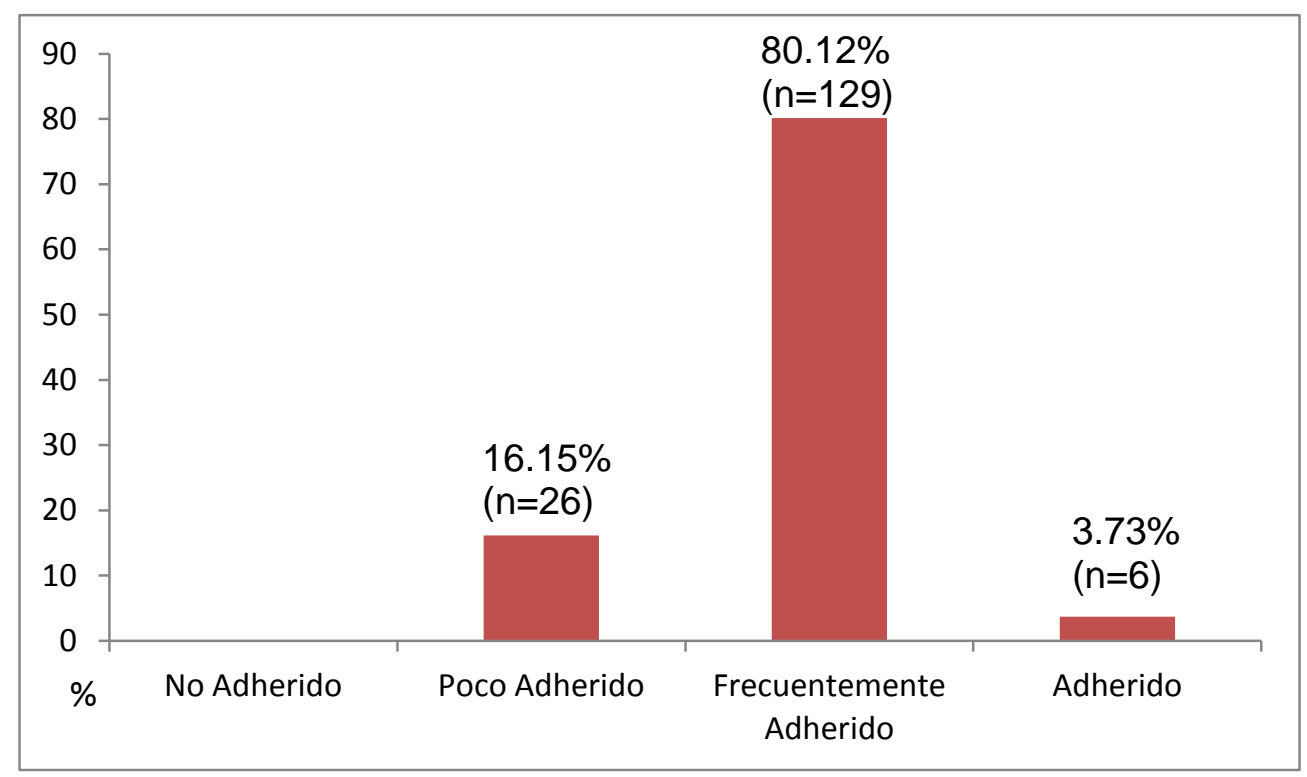

En la tabla 4, podemos observar el nivel de adherencia para cada indicador del instrumento

Tabla 4. Descripción de la Adherencia al Tratamiento Farmacológico y No Farmacológico en Pacientes con Falla Cardiaca $(n=161)$

\begin{tabular}{|c|c|c|c|c|c|}
\hline Indicadores & $\begin{array}{c}\text { Total de } \\
\text { Subindicador } \\
\text { es }\end{array}$ & $\begin{array}{l}\text { Rango } \\
\text { Escala } \\
\text { Likert }\end{array}$ & $\begin{array}{c}\text { Escala de } \\
\text { Calificació } \\
\mathbf{n}\end{array}$ & Interpretación & $\begin{array}{c}\text { Promedio de } \\
\text { Adherencia } \\
n=161\end{array}$ \\
\hline $\begin{array}{lll}\text { 1.Confía en } & \text { el } \\
\text { profesional de } & \text { la } \\
\text { salud } & & \end{array}$ & $\begin{array}{l}1 \\
2\end{array}$ & $\begin{array}{l}2.0-3.5 \\
3.6-5.5 \\
5.6-7.0 \\
7.1-8.0\end{array}$ & $\begin{array}{l}\text { Nunca } \\
\text { Muy poco } \\
\text { Frecuente } \\
\text { Siempre }\end{array}$ & $\begin{array}{l}\text { No adherente } \\
\text { Poca adherencia } \\
\text { Frecuentemente adherido } \\
\text { Adherido }\end{array}$ & $7.59(\mathrm{DE} \pm 0.7)$ \\
\hline $\begin{array}{l}\text { 2.Conocimiento del } \\
\text { tratamiento } \\
\text { farmacológico }\end{array}$ & $\begin{array}{l}3 \\
4 \\
5 \\
6\end{array}$ & $\begin{array}{r}4.0-7.0 \\
7.1-11.0 \\
11.1-15.0 \\
15.1-16.0\end{array}$ & $\begin{array}{l}\text { Nunca } \\
\text { Muy poco } \\
\text { Frecuente } \\
\text { Siempre }\end{array}$ & $\begin{array}{l}\text { No adherente } \\
\text { Poca adherencia } \\
\text { Frecuentemente adherido } \\
\text { Adherido }\end{array}$ & $9.27(\mathrm{DE} \pm 1.9)$ \\
\hline
\end{tabular}




\begin{tabular}{|c|c|c|c|c|c|}
\hline $\begin{array}{l}\text { 3. Información } \\
\text { sobre la posibilidad } \\
\text { de acceso a los } \\
\text { servicios }\end{array}$ & $\begin{array}{l}7 \\
8 \\
9\end{array}$ & $\begin{array}{r}3.0-5.5 \\
5.6-8.0 \\
8.1-11.0 \\
11.1-12.0\end{array}$ & $\begin{array}{l}\text { Nunca } \\
\text { Muy poco } \\
\text { Frecuente } \\
\text { Siempre }\end{array}$ & $\begin{array}{l}\text { No adherente } \\
\text { Poca adherencia } \\
\text { Frecuentemente adherido } \\
\text { Adherido }\end{array}$ & $9.44(\mathrm{DE} \pm 2.1)$ \\
\hline $\begin{array}{l}\text { 4. Aceptación del } \\
\text { diagnóstico dado } \\
\text { por el profesional } \\
\text { de la salud }\end{array}$ & $\begin{array}{l}10 \\
11 \\
12 \\
13\end{array}$ & $\begin{array}{r}4.0-7.0 \\
7.1-11.0 \\
11.1-15.0 \\
15.1-16.0\end{array}$ & $\begin{array}{l}\text { Nunca } \\
\text { Muy poco } \\
\text { Frecuente } \\
\text { Siempre }\end{array}$ & $\begin{array}{l}\text { No adherente } \\
\text { Poca adherencia } \\
\text { Frecuentemente adherido } \\
\text { Adherido }\end{array}$ & $13.34(\mathrm{DE} \pm 1.8)$ \\
\hline $\begin{array}{l}\text { 5. Reconocimiento } \\
\text { de signos y } \\
\text { síntomas }\end{array}$ & $\begin{array}{l}14 \\
15 \\
16\end{array}$ & $\begin{array}{r}3.0-5.5 \\
5.6-8.0 \\
8.1-11.0 \\
11.1-12.0\end{array}$ & $\begin{array}{l}\text { Nunca } \\
\text { Muy poco } \\
\text { Frecuente } \\
\text { Siempre }\end{array}$ & $\begin{array}{l}\text { No adherente } \\
\text { Poca adherencia } \\
\text { Frecuentemente adherido } \\
\text { Adherido }\end{array}$ & $6.85(\mathrm{DE} \pm 2.7)$ \\
\hline $\begin{array}{l}\text { 6.Busca refuerzo } \\
\text { externo para poder } \\
\text { cumplir con los } \\
\text { comportamientos } \\
\text { de salud }\end{array}$ & $\begin{array}{l}17 \\
18\end{array}$ & $\begin{array}{l}2.0-3.5 \\
3.6-5.5 \\
5.6-7.0 \\
7.1-8.0\end{array}$ & $\begin{array}{l}\text { Nunca } \\
\text { Muy poco } \\
\text { Frecuente } \\
\text { Siempre }\end{array}$ & $\begin{array}{l}\text { No adherente } \\
\text { Poca adherencia } \\
\text { Frecuentemente adherido } \\
\text { Adherido }\end{array}$ & $5.46(\mathrm{DE} \pm 1.3)$ \\
\hline $\begin{array}{l}\text { 7. Mantiene su peso } \\
\text { dentro del rango } \\
\text { recomendado }\end{array}$ & $\begin{array}{l}19 \\
20 \\
21 \\
22\end{array}$ & $\begin{array}{r}4.0-7.0 \\
7.1-11.0 \\
11.1-15.0 \\
15.1-16.0\end{array}$ & $\begin{array}{l}\text { Nunca } \\
\text { Muy poco } \\
\text { Frecuente } \\
\text { Siempre }\end{array}$ & $\begin{array}{l}\text { No adherente } \\
\text { Poca adherencia } \\
\text { Frecuentement adherido } \\
\text { Adherido }\end{array}$ & $10.88(\mathrm{DE} \pm 2.5)$ \\
\hline $\begin{array}{l}\text { 8.Conoce la dieta } \\
\text { recomendada }\end{array}$ & $\begin{array}{l}23 \\
24\end{array}$ & $\begin{array}{l}2.0-3.5 \\
3.6-5.5 \\
5.6-7.0 \\
7.1-8.0\end{array}$ & $\begin{array}{l}\text { Nunca } \\
\text { Muy poco } \\
\text { Frecuente } \\
\text { Siempre }\end{array}$ & $\begin{array}{l}\text { No adherente } \\
\text { Poca adherencia } \\
\text { Frecuentemente adherido } \\
\text { Adherido }\end{array}$ & $6.24(\mathrm{DE} \pm 1.1)$ \\
\hline $\begin{array}{lr}\text { 9. Realiza } & \text { el } \\
\text { ejercicio diario, } \\
\text { según } & \text { lo } \\
\text { recomendado por } \\
\text { el profesional de } \\
\text { salud }\end{array}$ & $\begin{array}{l}25 \\
26 \\
27\end{array}$ & $\begin{array}{r}3.0-5.5 \\
5.6-8.0 \\
8.1-11.0 \\
11.1-12.0\end{array}$ & $\begin{array}{l}\text { Nunca } \\
\text { Muy poco } \\
\text { Frecuente } \\
\text { Siempre }\end{array}$ & $\begin{array}{l}\text { No adherente } \\
\text { Poca adherencia } \\
\text { Frecuentemente adherido } \\
\text { Adherido }\end{array}$ & $9.88(\mathrm{DE} \pm 1.6)$ \\
\hline $\begin{array}{l}\text { 10.Estado de } \\
\text { ánimo apropiado } \\
\text { para su salud }\end{array}$ & $\begin{array}{l}28 \\
29 \\
30\end{array}$ & $\begin{array}{r}3.0-5.5 \\
5.6-8.0 \\
8.1-11.0 \\
11.1-12.0\end{array}$ & $\begin{array}{l}\text { Nunca } \\
\text { Muy poco } \\
\text { Frecuente } \\
\text { Siempre }\end{array}$ & $\begin{array}{l}\text { No adherente } \\
\text { Poca adherencia } \\
\text { Frecuentemente adherido } \\
\text { Adherido }\end{array}$ & $10.30(\mathrm{DE} \pm 1.5)$ \\
\hline
\end{tabular}

DE士=Desviación estándar.

También se midió el cumplimiento al tratamiento farmacológico con el test de MoriskyGreen, el $53.42 \%$ ( $n=86$; IC 95\% $45.39-61.30 \%)$ son cumplidores del tratamiento farmacológico; el $34.78 \%(n=56)$ se le olvida alguna vez tomar la medicación, el $59.63 \%(n=96)$ toma la medicación a la hora indicada, el $3.11 \%(n=5)$ cuando se encuentra bien, deja de tomar la medicación alguna vez y el 3.73\% $(n=6)$ si alguna vez se siente mal, deja de tomar la medicación.

\section{DISCUSIÓN}

Se determinó un promedio de adherencia de 89.3 puntos en los pacientes atendidos en la CFCTC, lo cual indica que los pacientes se encuentran frecuentemente adheridos. Estos resultados son similares a los informados en el estudio de Achury ${ }^{9}$ en Bogotá quien presentó una adherencia promedio de 89.4 puntos, posterior a la aplicación de un plan educativo que fomenta el autocuidado. Las similitudes de la media de prevalencia de adherencia podrían explicarse por las características de la población incluida en ese estudio; el promedio de edad fue de 68 años, el $54 \%$ eran hombres, el $76 \%$ solo tenían educación básica primaria, la clase funcional New York Heart Association (NYHA) el 6\% eran clase I, el $66 \%$ clase II y el $28 \%$ clase III. En 
nuestra población el promedio de edad fue de 64 años, el $60.25 \%$ eran hombres, el $50.93 \%$ tenían menos o igual a 6 años cursados y aprobados y la proporción de la clase funcional NYHA eran similares.

En cuanto a la confianza en el profesional de la salud, la media de la adherencia indica que se encuentran adheridos. Heszen y Lapinska ${ }^{15}$, determinaron que una actitud positiva entre la interacción médico-paciente favorece el cumplimiento de las órdenes médicas en los pacientes. En una investigación cualitativa, encontraron que una relación caracterizada por la confianza entre el paciente y el proveedor era un factor de motivación para mantener un alto nivel de adherencia ${ }^{16}$.

Con respecto al conocimiento del tratamiento farmacológico, la media mostró un nivel de poca adherencia. El conocimiento va muy de la mano con el nivel educativo, varios estudios han relacionado consistentemente una mayor adherencia con un nivel educativo alto ${ }^{17,18}$, en nuestro estudio la mediana de años cursados y aprobados fue de 6 años, lo que podría estar explicando la poca adherencia en este indicador.

En el indicador información sobre la posibilidad de acceso a los servicios de salud los pacientes se encuentran frecuentemente adheridos. Basterra ${ }^{19}$ afirma que el fácil acceso a los servicios sanitarios, la reducción al máximo de los tiempos de espera, la concertación previa y planificada de las citas o incluso la asistencia en el propio domicilio del paciente, han demostrado tener una influencia positiva en el grado de cumplimiento.

La aceptación del diagnóstico dado por el profesional de la salud, los pacientes se encuentran frecuentemente adheridos. Salinas y $\mathrm{Nava}^{20}$ concluyen que algunas causas de incumplimiento terapéutico se deben a no aceptar el diagnóstico, la negación de la enfermedad, la estigmatización entre otras.

El promedio del indicador reconocimiento de signos y síntomas se encuentra en un nivel de poca adherencia, mientras que Achury ${ }^{9}$ encontró que los pacientes en cuanto a este indicador se encuentran frecuentemente adheridos. Esto podría explicarse por las poblaciones estudiadas (paciente hospitalizados versus pacientes ambulatorios), los pacientes hospitalizados recuerdan más los signos y síntomas de la falla cardiaca, mientras los pacientes de la clínica de falla cardiaca son pacientes controlados con pocas o casi ninguna hospitalización en el último año.

A pesar que el $89.44 \%$ de los pacientes cuentan con un soporte de cuidador y realizan sus controles en un clínica de falla cardiaca, el indicador busca refuerzo externo para poder cumplir con los comportamiento de salud su nivel es poco adherente. La clínica de falla cardiaca cuenta con un equipo interdisciplinario que ofrece periódicamente capacitaciones a sus pacientes, sin embargo, hay razones por las cuales los pacientes no pueden asistir a dichas capacitaciones: no tienen una persona que los acompañe, viven en otras ciudades y todo lo que esto implica, desplazamiento, tiempo, recursos económicos entre otros.

El indicador mantiene su peso dentro del rango recomendado, los pacientes demuestran poca adherencia. Este indicador evalúa el control de peso diariamente, la ausencia de edemas, el conocimiento de la cantidad de agua que debe consumir diariamente y el realizar un balance de ingesta y eliminación de líquidos diariamente.

En cuanto al indicador 8 y 9, que hace referencia a la dieta y actividad física los pacientes se encuentran frecuentemente adheridos. Es decir los pacientes conocen y 
llevan una dieta rica en frutas, verduras y baja en sal y grasas, realizan la actividad física recomendada por el profesional e identifican los signos de cese de actividad física.

Con respecto al estado de ánimo apropiado para su salud los pacientes se encuentran frecuentemente adheridos. En una investigación cuantitativa, los pacientes que creían que podrían mejorar o tener una buena calidad de vida como resultado de tomar sus medicamentos estaban más dispuestos a cumplir con el régimen prescrito ${ }^{21}$.

Una de las posibles limitaciones que tiene el presente estudio es la capacidad de generalizar sus resultados, debido a que el tipo de muestreo llevado a cabo fue no probabilístico, sin embargo la metodología realizada, favorecen la validez interna del mismo. La adherencia fue medida por autoinforme, lo cual podría estar sobreestimando los resultados de la adherencia al tratamiento, como ha sido sugerida por otros autores ${ }^{22}$. Sin embargo, el instrumento de evaluación de adherencia al tratamiento farmacológico y no farmacológico en los pacientes con falla cardiaca, ya ha sido validado previamente, mostrando un buen desempeño como herramienta de diagnóstico de adherencia y también se tomaron medidas para evitar los sesgos de información.

\section{CONCLUSIONES}

Los resultados del presente estudio son un acercamiento a la problemática de la adherencia al tratamiento farmacológico y no farmacológico de los pacientes con falla cardiaca. En el presente estudio con respecto a la adherencia global al tratamiento farmacológico y no farmacológico, la mayoría de los pacientes de la CFCTC se encuentran frecuentemente adheridos al régimen terapéutico. La mitad de los pacientes son cumplidores del tratamiento farmacológico con el test de MoriskyGreen.

Los pacientes demostraron frecuentemente adherencia a seis indicadores: confía en el profesional de salud; información sobre la posibilidad de acceso a los servicios; aceptación del diagnóstico dado por el profesional; conoce la dieta recomendada; realiza el ejercicio diario según lo recomendado por el profesional de salud y estado de ánimo apropiado para su salud.

Se identificó poca adherencia a cuatro indicadores: conocimiento del tratamiento farmacológico; reconocimiento de signos y síntomas; busca refuerzo externo para poder cumplir con los comportamientos de salud y mantiene su peso dentro del rango recomendado.

Se recomienda para futuras investigaciones, la realización de estudios multicéntricos con muestreos probabilísticos, con el fin de obtener resultados generalizables a todos los pacientes con falla cardiaca de nuestro país atendidos dentro de un programa 0 Clínica de Falla Cardiaca, con el fin de direccionar acciones encaminadas a mejorar o mantener los niveles de adherencia y logrando así mejor la calidad de vida de los pacientes que viven con esta enfermedad. 


\section{Agradecimientos}

Al Instituto Colombiano de Ciencia y Tecnología COLCIENCIAS y el programa Jóvenes Investigadores e Innovadores 2010 "Virginia Gutiérrez Pineda", al grupo de Investigación de Enfermería GRINFER de la Universidad Industrial de Santander y a la Clínica de Falla Cardiaca y Trasplante Cardiaco de la Fundación Cardiovascular.

\section{BIBLIOGRAFÍA}

1. McMurray JJ, Pfeffer MA. Heart failure. Lancet. 2005;365(9474):1877-1889.

2. Davis RC, Hobbs FD, Lip GY. ABC of heart failure. History and epidemiology. BMJ. 2000;320(7226):39-42.

3. Bui AL, Horwich TB, Fonarow GC. Epidemiology and risk profile of heart failure. Nat Rev Cardiol. 2011;8(1):30-41.

4. Heydari A, Ahrari S, Vaghee S. The relationship between self-concept and adherence to therapeutic regimens in patients with heart failure. J Cardiovasc Nurs. 2011;26(6):475-480.

5. Maeda U, Shen BJ, Schwarz ER, Farrell KA, Mallon S. Self-Efficacy mediates the associations of social support and depression with treatment adherence in heart failure patients. Int J Behav Med. 2013:20(1):88-96.

6. Sabaté E. Adherencia a los tratamientos a largo plazo: pruebas para la acción. Organización Mundial de la Salud (OMS) 2004; traducción de la Organización Panamericana de la Salud (OPS).

7. Wu JR, Moser DK, Lennie TA, Burkhart PV. Medication adherence in patients who have heart failure: a review of the literature. Nurs Clin North Am. 2008;43(1):133153.

8. Libertad MA. Repercusiones para la salud pública de la adherencia terapéutica deficiente. Rev Cubana Salud Pública. 2006;32(3).

9. Achury Saldaña DM. Autocuidado y adherencia en pacientes con falla cardiaca. Aquichan. 2007;7(2):139-160.

10. CDC. Epi Info, v. 6.04d - StatCalc. Epidemiología en ordenadores. Atlanta, Georgia. Enero, 2001.

11. Achury Saldaña DM, Sepúlveda Carrillo GJ, Rodríguez Colmenares SM, Giraldo IC. Validez y confiabilidad de un instrumento evaluativo de adherencia en pacientes con falla cardiaca. Enfermería Global. 2012;26:1-9.

12. García Pérez AM, Leiva Fernández F, Martos Crespo F, García Ruíz AJ, Prados Torres D, Sánchez de la Cuesta, et al. ¿Cómo diagnosticar el cumplimiento terapéutico en atención primaria? Medicina de Familia (And) 2000;(1):13-19.

13. Epidata Association. Epidata Software 3.1. 2004; Dinamarca.

14. StataCorp. Stata Statistical Sofware: Release 12.0. Collage Station, Texas: Stata Corporation. 2003.

15. Heszen-Klemens I, Lapinska E. Doctor-patient interaction, patients' health behavior and effects of treatment. Soc Sci Med. 1984;19(1):9-18.

16. Simpson SH, Farris KB, Johnson JA, Tsuyuki KB. Using focus groups to identify barriers to drug use in patients with congestive heart failure. Pharmacotherapy 2000;20(7):823-829.

17. Evangelista LS, Berg J, Dracup K. Relationship between psychosocial variables and compliance in patients with heart failure. Heart Lung. 2001;30(4):294-301.

18. Rockwell JM, Riegel B. Predictors of self-care in persons with heart failure. Heart Lung. 2001;30(1):18-25.

19. Basterra M. El cumplimiento terapéutico. Pharmaceuticall Care. 1999;1:97-106. 
20. Salinas Cruz E, Nava Galán MG. Adherencia terapéutica. Enf Neurol(Mex). 2012;11(2):102-104.

21. Hicks FD, Holm K. Self-management decision influences in heart failure: a preliminary investigation. Clin Nurs Res. 2003;12(1):69-84.

22. Nogués Solán X, Sorli Redó ML, Villar García J. Instrumentos de medida de adherencia al tratamiento. An. Med. Interna. 2007;24(3):138-41. 